\title{
Calcium chelator BAPTA-AM protects against iron overload-induced chondrocyte mitochondrial dysfunction and cartilage degeneration
}

\author{
XINGZHI JING ${ }^{1 *}$, QIANG WANG ${ }^{2 *}$, TING DU ${ }^{3}$, WEIMIN ZHANG ${ }^{1}$, XIAOYANG LIU ${ }^{1}$,

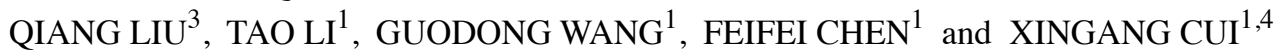 \\ Departments of ${ }^{1}$ Spine Surgery and ${ }^{2}$ Human Resources, Shandong Provincial Hospital Affiliated to \\ Shandong First Medical University, Jinan, Shandong 250021; ${ }^{3}$ Yidu Cloud (Beijing) Technology Co., Ltd., Beijing 100191; \\ ${ }^{4}$ Department of Spine Surgery, Shandong Provincial Hospital, Cheeloo College of Medicine, \\ Shandong University, Jinan, Shandong 250021, P.R. China
}

Received May 7, 2021; Accepted August 11, 2021

DOI: $10.3892 /$ ijmm.2021.5029

\begin{abstract}
Osteoarthritis (OA) is a common joint disease that is characterized by cartilage degradation. Iron deposition in the joints is common during the pathogenic progression of $\mathrm{OA}$ and recent studies have indicated that iron overload is an important contributor to OA progression. Calcium chelators have been reported to inhibit iron influx via modulating transferrin receptor protein 1 internalization, and they have been identified as a potential approach to the treatment of iron overload-induced diseases. The aim of the present study was to investigate the effect of calcium chelators on the progression of iron overload-induced OA. Primary chondrocytes were treated with various concentrations of ferric ammonium citrate (FAC) to mimic iron overload in vitro, followed by co-treatment with the calcium chelator BAPTA acetoxymethyl ester (BAPTA-AM). Subsequently, intracellular iron levels, cell viability, reactive oxygen species (ROS) levels, mitochondrial function and morphological changes, as well as MMP levels, were detected using commercial kits. It was demonstrated that FAC treatment significantly promoted chondrocyte apoptosis and the expression of MMPs, and these effects were reversed by co-treatment with BAPTA-AM. Moreover, BAPTA-AM suppressed iron influx into chondrocytes and inhibited iron overload-induced ROS production and mitochondrial dysfunction. These results indicated that calcium chelators
\end{abstract}

Correspondence to: Professor Xingang Cui, Department of Spine Surgery, Shandong Provincial Hospital, Cheeloo College of Medicine, Shandong University, 324 Jingwu Weiqi Road, Jinan, Shandong 250021, P.R. China

Email: spine2014@163.com

*Contributed equally

Key words: iron overload, calcium chelator, osteoarthritis, BAPTA acetoxymethyl ester, mitochondrial dysfunction may be of value in the treatment of iron metabolism-related diseases and iron overload-induced OA progression.

\section{Introduction}

Our previous studies have demonstrated the detrimental effect of iron on osteoarthritis (OA) chondrocytes (1), and that arresting iron influx or decreasing iron concentration using iron chelators may inhibit iron deposition-induced OA progression (2). Iron chelators are drugs that may be beneficial for patients with iron overload (3). However, considering the severe side effects of iron chelators, such as renal damage and neurological dysfunction, new strategies for inhibiting the iron influx into cells must be investigated (4). As the most abundant trace metal in the human body, iron is crucial for the majority of cellular metabolic processes (5). Iron homeostasis is delicately regulated in humans, and iron overload may damage cells by promoting the production of reactive oxygen species (ROS) (6). Iron overload can generate highly toxic hydroxyl radicals and result in ferroptosis (7). As the human body lacks effective ways for excreting excess iron, iron overload is common among elderly individuals and has been reported to be implicated in several diseases or pathological conditions (8). OA is one of the most common complications in a variety of diseases characterized by abnormal intracartilaginous hemorrhage or iron deposition, such as hereditary hemochromatosis, hemophilic arthropathy and rheumatoid arthritis, in which cartilage degeneration and $\mathrm{OA}$ progression are common. Moreover, iron deposition has also been observed in traumatic and age-related OA (9). Iron is considered to be the main cause of continual degeneration of the knee joint in traumatic arthritis (10). Iron has also been reported to induce crystal deposition in the supersaturated synovial fluid through the process of nucleation and cartilage damage in OA (11). Moreover, recent findings suggested that cellular iron overload-induced oxidative stress and mitochondrial dysfunction play pivotal roles in OA progression $(12,13)$. Oxidative stress and mitochondrial dysfunction were also reported to play important roles during OA development (14). Iron metabolism has been demonstrated 
to be an important contributor to normal mitochondrial function. By converting from $\mathrm{Fe}^{2+}$ to $\mathrm{Fe}^{3+}$ and vice versa, iron can participate in the mitochondrial oxidative respiratory chain and exchange a single electron with other substrates. Excess iron can lead to increased ROS production and destroy mitochondrial structure; mitochondrial dysfunction, in turn, can produce excess ROS and result in MMP activation and decreased collagen synthesis (15).

Iron in the diet is taken up by enterocytes through the divalent metal transporter 1 (DMT1) and exported via ferroportin to the general circulation. Then, plasma iron is delivered into tissues or organs via transferrin (TF) receptor 1 (TfR1)-mediated endocytosis after binding to TF in most types of cells (16). As $\mathrm{Ca}^{2+}$ and $\mathrm{Fe}^{2+}$ have equally positive charges, it is possible that $\mathrm{Ca}^{2+}$ may affect $\mathrm{Fe}^{2+}$ influx. It was recently demonstrated that the L-type and T-type calcium channels are involved in iron uptake into the heart under conditions of iron overload (17). Sripetchwandee et al (18) reported that blockade of the mitochondrial calcium uniporter prevents cardiac mitochondrial dysfunction caused by iron overload. Kumfu et al (19) found that combining an iron chelator and a T-type calcium channel blocker exhibited greater cardioprotective efficacy compared with iron chelator alone in an iron overload-induced thalassemia mouse model. Moreover, Zhang et al (20) reported that calcium channel blockers (CCBs) ameliorated iron overload-associated hepatic fibrosis by altering iron transport, and indicated that CCBs are potential therapeutic agents for iron overload-induced diseases. Calcium was reported to modulate receptor-mediated endocytosis and, as TfR1 modulates iron influx through this pathway, it was further demonstrated that calcium could antagonize iron-TF-TfR1 complex internalization and, thus, modulate iron uptake (21).

The present study was undertaken to investigate the effect of the calcium chelator BAPTA acetoxymethyl ester (BAPTA-AM) on iron overload-induced chondrocyte apoptosis and MMP expression. Primary chondrocytes were treated with various concentrations of ferric ammonium citrate (FAC) to mimic iron overload in vitro, and the effects of BAPTA-AM on iron influx into chondrocytes, intracellular iron concentration and ROS production were investigated. Moreover, mitochondrial depolarization and mitochondrial morphology were examined to determine the mitochondrial function using immunofluorescence and flow cytometry assays. The aim was to determine whether calcium chelators may be potential therapeutic agents in treating iron metabolism diseases and iron overload-induced OA progression.

\section{Materials and methods}

Cell isolation and culture. A total of 30 male C57/BL6 mice (aged 5 days and weighing $<5 \mathrm{~g}$ ) were used in the present study. The mice were anesthetized by intraperitoneal injection of $2 \%$ pentobarbital sodium ( $35 \mathrm{mg} / \mathrm{kg}$ body weight) and sacrificed via cervical dislocation. All animal protocols were approved by the Institutional Animal Care of the Shandong Provincial Hospital Affiliated to Shandong First Medical University (no. 2020-526). Briefly, cartilage was obtained from bilateral knee joints and minced into pieces. The cartilage pieces were then washed with cold PBS, followed by the addition of
$0.25 \%$ trypsin-EDTA for $30 \mathrm{~min}$ at $37^{\circ} \mathrm{C}$. After washing with PBS, samples were digested with $0.25 \%$ collagenase solution at $37^{\circ} \mathrm{C}$ for $7 \mathrm{~h}$. After collection and centrifugation at $100 \mathrm{x} \mathrm{g}$ for $10 \mathrm{~min}$ at $37^{\circ} \mathrm{C}$, chondrocytes were resuspended in DMEM/F12 (HyClone; Cytiva) supplemented with 10\% FBS (Gibco; Thermo Fisher Scientific, Inc.) and cultured in a humidified atmosphere of $5 \% \mathrm{CO}_{2}$ at $37^{\circ} \mathrm{C}$. Chondrocytes at passages 1 or 2 were used in the following experiments.

Cell Counting Kit-8 (CCK-8) assay. CCK-8 was obtained from Wuhan Boster Biological Technology, Ltd. and used to detect cell viability according to the manufacturer's instructions. Briefly, cells were collected and resuspended in DMEM/F12 supplemented with $10 \%$ FBS at a density of $5 \times 10^{4}$ cells $/ \mathrm{ml}$, and $200 \mu \mathrm{l}$ medium containing $1 \times 10^{4}$ cells were added into each well of a 96-well plate. Increasing concentrations of FAC (1-200 $\mu \mathrm{m})$ were then added and cultured for different time periods (0-5 days). The culture medium was changed every other day. CCK-8 solution $(10 \mu \mathrm{l})$ was added into each well on days $0,1,3$ and 5 and incubated at $37^{\circ} \mathrm{C}$ in the dark for $1 \mathrm{~h}$. The absorbance at $450 \mathrm{~nm}$ was recorded using spectral IMAX190 absorbance microplate reader (Molecular Devices, LLC). Optical density values at $450 \mathrm{~nm}$ were recorded to evaluate cell viability.

Western blot analysis. Chondrocytes were seeded in 6-well plates at a density of $1 \times 10^{5}$ cells per well. After being subjected to various treatments, chondrocytes were collected and lysed with $100 \mu$ l RIPA lysis buffer (Wuhan Boster Biological Technology, Ltd.) on ice for $1 \mathrm{~h}$. The lysates were then collected and centrifuged at $300 \mathrm{x} \mathrm{g}$ for $20 \mathrm{~min}$ at $4^{\circ} \mathrm{C}$, the supernatant was collected and the protein concentration was detected using the BCA assay method. Equal amounts of protein ( $25 \mu \mathrm{g} / \mathrm{lane})$ were electrophoresed using 12\% SDS-PAGE and transferred to PVDF membranes (MilliporeSigma). The PVDF membranes were then blocked with $5 \%$ skimmed milk for $1 \mathrm{~h}$ at room temperature and incubated with targeted primary antibodies against MMP3 (1:1,000; cat. no. 17873-1-AP; ProteinTech Group, Inc.), MMP13 (1:1,000; cat. no. ab39012; Abcam), mitochondrial fission 1 protein (FIS1; 1:1,000; cat. no. 10956-1-AP; ProteinTech Group, Inc.), dynamin-related protein 1 (DRP1; 1:1,000; cat. no. 8570; Cell Signaling Technology, Inc.), mitochondrial fission factor (MFF; 1:1,000; cat. no. 84580; Cell Signaling Technology, Inc.), BAX (1:1,000; cat. no. 50599-2-Ig; ProteinTech Group, Inc.), cytochrome $c$ (1:1,000; cat. no. ab13575; Abcam) and $\beta$-actin (1:500; cat. no. BM0627; Wuhan Boster Biological Technology, Ltd.) at $4^{\circ} \mathrm{C}$ overnight. After washing with Tris-buffered saline with Tween-20 [50 mM Tris ( $\mathrm{pH} 7.6), 150 \mathrm{~mm} \mathrm{Nacl}$ and $0.1 \%$ Tween-20] three times, the membranes were incubated with a horseradish peroxidase-conjugated secondary antibody $(1: 2,000$; cat. no. BA1055; Wuhan Boster Biological Technology, Ltd.) at room temperature for $1 \mathrm{~h}$. The protein bands were visualized with enhanced chemiluminescence reagent (Wuhan Boster Biological Technology, Ltd.) and images were captured. The density of each band was quantified by ImageJ software (version 1.8.0; National Institutes of Health).

Reverse transcription-quantitative $(R T-q) P C R$ analysis. Chondrocytes were seeded in 12 -well plates at a density 
of $5 \times 10^{4}$ cells per well and treated with $100 \mu \mathrm{m} \mathrm{FAC}$ (MilliporeSigma) for $24 \mathrm{~h}$. Chondrocytes were then treated with TRIzol ${ }^{\circledR}$ (Invitrogen; Thermo Fisher Scientific, Inc.) and total RNA was extracted using the Toyobo Total RNA Extraction kit (Toyobo Life Science) following the manufacturer's instructions. Complementary DNA (cDNA) was synthesized from RNA using the First Strand cDNA Synthesis kit (Toyobo Life Science) and amplified using SYBR Green Real-time PCR Master Mix (Toyobo Life Science) under the following cycling conditions: $30 \mathrm{sec}$ of polymerase activation at $95^{\circ} \mathrm{C}$, followed by 40 cycles at $95^{\circ} \mathrm{C}$ for $5 \mathrm{sec}$ and $60^{\circ} \mathrm{C}$ for $30 \mathrm{sec}$. Relative expression levels were calculated using the $2^{-\Delta \Delta \mathrm{cq}}$ method (22). GAPDH was used as an internal control. Each cDNA sample was assayed in triplicate. The sequences of the primers for the genes of interest are listed as follows: A disintegrin and metalloproteinase with thrombospondin motifs 5 (ADAMTS5): Forward, 5'-TATGACAAGTGCGGA GTATG-3' and reverse, 5'-TTCAGGGCTAAATAGGCA GT-3'; MMP3: Forward, 5'-ATGCCCACTTTGATGATG ATGAAC-3' and reverse, 5'-CCACGCCTGAAGGAAGAG ATG-3'; MMP13: Forward, 5'-GCTGGACTCCCTGTTG-3' and reverse, 5'-TCGGAGCCTGTCAACT-3'; and GAPDH: Forward, 5'-CTCCCACTCTTCCACCTTCG-3' and reverse, 5'-TTGCTGTAGCCGTATTCATT-3'.

Immunofluorescence staining. Chondrocytes at passage 1 or 2 were seeded in 24-well plates at a density of $1 \times 10^{4}$ cells per well. Following treatment with $100 \mu \mathrm{m}$ FAC (MilliporeSigma) for $24 \mathrm{~h}$, cells were washed with serum-free DMEM/F12 (HyClone; Cytiva) and incubated with diluted Mito-Tracker Red solution (1:1,000; Invitrogen; Thermo Fisher Scientific, Inc.) for $30 \mathrm{~min}$ at $37^{\circ} \mathrm{C}$ in the dark. Cells were then washed with PBS three times and fixed with $4 \%$ paraformaldehyde for $20 \mathrm{~min}$ at room temperature. Cells were then washed with PBS three times and treated with $0.1 \%$ Triton $\mathrm{X}-100$ for $15 \mathrm{~min}$. After blocking with 10\% FBS (Wuhan Boster Biological Technology, Ltd.) for $30 \mathrm{~min}$ at room temperature, the cells were then incubated with mixed rabbit polyclonal BAX primary antibody (1:1,000; cat. no. 50599-2-Ig; ProteinTech Group, Inc.) and mouse monoclonal cytochrome $c$ antibody (1:1,000; cat. no. ab13575; Abcam) at $4^{\circ} \mathrm{C}$ overnight. Following rinsing with $\mathrm{PBS}$ three times, mixed goat anti-rabbit FITC-conjugated secondary antibody (1:200; cat. no. BA1105; Wuhan Boster Biological Technology, Ltd.) and goat anti-mouse Dylight 405 secondary antibody (1:400; Beyotime Institute of Biotechnology) was added and incubated for $1 \mathrm{~h}$ at room temperature in the dark. Finally, cells were washed with PBS and analyzed at a magnification of x200 under a fluorescence microscope (EVOS ${ }^{\mathrm{TM}}$ FL Auto; Thermo Fisher Scientific, Inc.).

Measurement of intracellular iron levels. Briefly, primary chondrocytes were treated with $100 \mu \mathrm{m} \mathrm{FAC,} \mathrm{with} \mathrm{or} \mathrm{without}$ $10 \mu \mathrm{m}$ BAPTA-AM (Selleck Chemicals), for $6 \mathrm{~h}$. Then, cells were loaded with $0.5 \mu \mathrm{mol} / 1$ calcein-AM for $30 \mathrm{~min}$ at $37^{\circ} \mathrm{C}$. Chondrocytes were then washed with PBS three times. Free calcein (non-metal-bound) was measured for fluorescence (excitation: $488 \mathrm{~nm}$; emission: $517 \mathrm{~nm}$ ) using a fluorescence microscope (EVOS ${ }^{\mathrm{TM}}$ FL Auto; Thermo Fisher Scientific, Inc.) at a magnification of $\mathrm{x} 200$. To quantify the fluorescence values, four separate fields monitored were randomly selected and the mean fluorescence signal was processed with Image Pro Plus software (version 6.0; National Institutes of Health).

Evaluation of intracellular ROS. Chondrocytes were seeded in 12-well plates at a density of $1 \times 10^{4}$ cells per well and treated with $100 \mu \mathrm{m} \mathrm{FAC}$, with or without $10 \mu \mathrm{m}$ BAPTA-AM, for $24 \mathrm{~h}$. After treatment, the intracellular ROS level was determined using the Reactive Oxygen Species Assay kit (cat. no. S0033; Beyotime Institute of Biotechnology) according to manufacturer's instructions. Briefly, chondrocytes were washed with serum-free DMEM/F12 (HyClone; Cytiva) three times, then $10 \mu \mathrm{m}$ dichloro-dihydro-fluorescein diacetate (DCFH-DA) was added to the culture medium and incubated in the dark for $20 \mathrm{~min}$. Chondrocytes were then washed with DMEM/F12 (HyClone; Cytiva) and examined at a magnification of x100 under a fluorescence microscope (EVOS ${ }^{\mathrm{TM}}$ FL Auto; Thermo Fisher Scientific, Inc.) at an excitation wavelength of $488 \mathrm{~nm}$ and an emission wavelength of $525 \mathrm{~nm}$.

Measurement of mitochondrial membrane potential. Cells were seeded in 12-well plates at a density of $1 \times 10^{4}$ per well and treated with $100 \mu \mathrm{m}$ FAC, with or without $10 \mu \mathrm{m}$ BAPTA-AM, for $24 \mathrm{~h}$. After washing with DMEM/F12 three times, chondrocytes were incubated with JC-1 staining solution (Beyotime Institute of Biotechnology) for $30 \mathrm{~min}$ at room temperature. After incubation, the culture medium was changed and the chondrocytes were washed with washing buffer. The fluorescence was examined using a fluorescence microscope. Aggregated JC-1 in mitochondria with high mitochondrial membrane potential levels produced red fluorescence, whereas when mitochondrial membrane potential decreased, JC-1 was found in its monomer form and produced green fluorescence.

To quantify mitochondrial membrane potential in chondrocytes, cells were incubated with JC-1 staining solution as described above. After incubation, cells were re-suspended in $500 \mathrm{ml} \mathrm{DMEM} / \mathrm{F} 12$ and the fluorescence intensity was recorded with a FACSCalibur flow cytometer (BD Biosciences).

Mitochondrial-specific fluorescence staining. Mito-Tracker Green (Beyotime Institute of Biotechnology) was used to evaluate the morphological changes of mitochondria. Mito-Tracker Green is an mitochondrial membrane potential-independent mitochondrial staining reagent. Briefly, chondrocytes were treated with FAC $(100 \mu \mathrm{m})$, with or without $10 \mu \mathrm{m}$ BAPTA-AM, for $24 \mathrm{~h}$; subsequently, cells were washed twice with FBS-free DMEM/F12 and incubated with diluted Mito-Tracker Green solution $(1: 1,000)$ for $30 \mathrm{~min}$ at $37^{\circ} \mathrm{C}$ in the dark. The shapes of the mitochondria were imaged at a magnification of $x 400$ under a fluorescence microscope $\left(\right.$ EVOS $^{\mathrm{TM}}$ FL Auto; Thermo Fisher Scientific, Inc.).

Evaluation of apoptosis. An Annexin V-FITC/PI Apoptosis Detection kit (cat. no. C1063; Beyotime Institute of Biotechnology) was used to detect the cell apoptosis rate according to the manufacturer's instructions. Chondrocytes were seeded in 6 -well plates at a density of $1 \times 10^{5}$ per well and treated with $100 \mu \mathrm{m}$ FAC, with or without $10 \mu \mathrm{m}$ BAPTA-AM, for $24 \mathrm{~h}$. After the aforementioned treatment, cells were washed with PBS and then stained with 
A

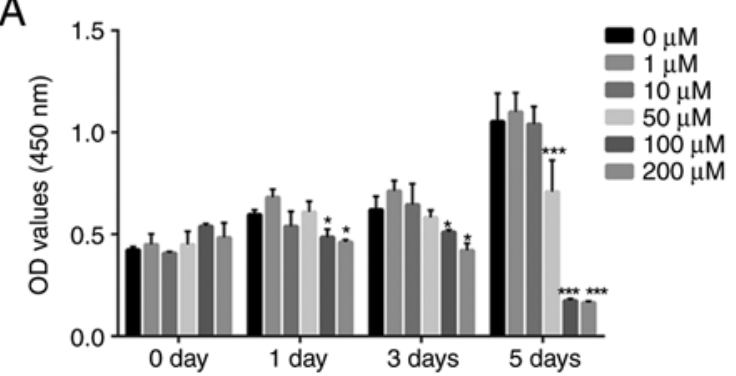

C

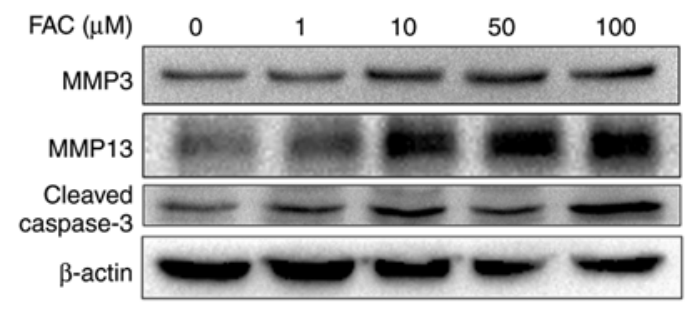

B
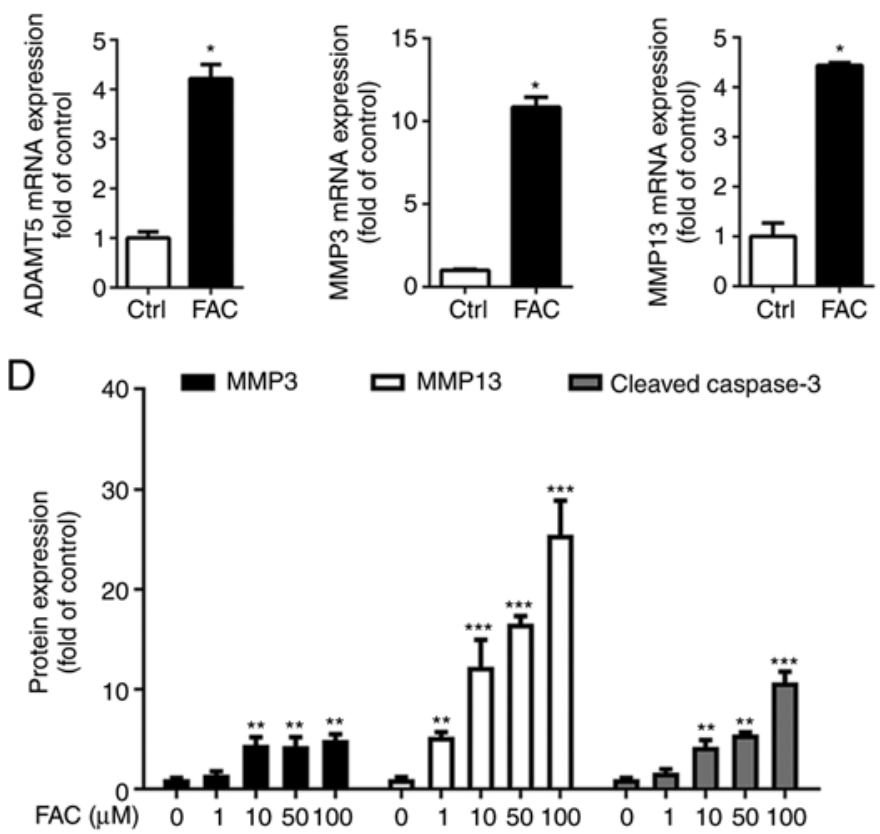

Figure 1. Iron overload promotes chondrocyte apoptosis and osteoarthritis-related marker expression. (A) Chondrocytes were treated with various concentrations of FAC $(0,1,10,50,100$ and $200 \mu \mathrm{m})$ for different time periods (0, 1, 3 and 5 days). Cell viability was determined using the Cell Counting Kit- 8 assay. Data are presented as mean $\pm \mathrm{SD}$. ${ }^{*} \mathrm{P}<0.05 ;{ }^{* * *} \mathrm{P}<0.001$ vs. $0 \mu \mathrm{M}$ FAC. (B) Chondrocytes were treated with $100 \mu \mathrm{m}$ FAC for $24 \mathrm{~h}$ and reverse transcriptionquantitative PCR analysis was conducted to examine ADAMTS5, MMP3 and MMP13 gene expression. Data are presented as mean \pm SD. * $<0.05$ vs. Ctrl. (C and D) Chondrocytes were treated with increasing concentrations of FAC for $24 \mathrm{~h}$ and western blotting was conducted to examine MMP3, MMP13 and cleaved caspase-3 protein expression. The band density of MMP3, MMP13 and cleaved caspase-3 was quantified and normalized to control. Data are presented as mean \pm SD. ${ }^{* *} \mathrm{P}<0.01 ;{ }^{* * *} \mathrm{P}<0.001$ vs. $0 \mu \mathrm{M}$ FAC. ADAMTS5, A disintegrin and metalloproteinase with thrombospondin motifs 5; FAC, ferric ammonium citrate; Ctrl, control group; OD, optical density.

Annexin V-FITC/PI for $20 \mathrm{~min}$ in the dark. A FACSCalibur flow cytometer (BD Biosciences) was used to analyze the results. Annexin $\mathrm{V}^{+} / \mathrm{PI}^{-}$and Annexin $\mathrm{V}^{+} / \mathrm{PI}^{+}$cells were considered as early and late apoptotic cells, respectively.

Statistical analysis. The results are presented as mean \pm SD. All experiments were repeated three times independently. Unpaired Student's t-test was used to evaluate differences between two groups. Differences among multiple groups were determined using one-way analysis of variance followed by Tukey's post hoc test. $\mathrm{P}<0.05$ was considered to indicate statistically significant differences.

\section{Results}

Iron overload promotes chondrocyte apoptosis and MMP expression. First, primary chondrocytes were treated with various concentrations of FAC to mimic iron overload in vitro, and then CCK-8 assay was performed to verify the cytotoxic effects of iron on chondrocytes. As shown in Fig. 1A, FAC decreased chondrocyte viability in a dose-dependent manner. FAC at a concentration of $1 \mu \mathrm{m}$ slightly enhanced chondrocyte proliferation, while FAC at concentrations $>50 \mu \mathrm{m}$ significantly decreased chondrocyte viability. Western blotting demonstrated that FAC promoted the expression of the cell apoptosis marker cleaved caspase-3 in a dose-dependent manner, and FAC at $100 \mu \mathrm{m}$ was associated with significantly elevated cleaved caspase-3 expression. Next, the expression of the OA-related markers, MMP3 and MMP13, was examined using western blot and RT-qPCR analyses. As shown in Fig. 1C and D, FAC promoted MMP3 and MMP13 protein expression in a dose-dependent manner, with the protein levels peaking at $100 \mu \mathrm{m}$ FAC. Similar results were obtained using RT-qPCR analysis, with $100 \mu \mathrm{m}$ FAC significantly promoting ADAMTS5, MMP3 and MMP13 mRNA expression (Fig. 1B).

Iron overload impairs mitochondrial dynamics in chondrocytes. Mitochondrial function plays an important role in OA progression (15). To further investigate the mechanisms underlying iron overload-induced accelerated progression of $\mathrm{OA}$, chondrocytes at passage 1 or 2 were stimulated with FAC to mimic iron overload in vitro. Immunofluorescence staining was then performed to examine the localization of cytochrome $c$ and BAX proteins. It was observed that FAC promoted mitochondrial BAX expression and translocation of cytochrome $c$ from the mitochondria to the cytoplasm, indicating that mitochondrial structure was destroyed after FAC treatment (Fig. 2A). Mitochondrial fission and fusion play important roles in maintaining the function and morphology of mitochondria (23). The results of the present study demonstrated that the expression of mitochondrial fission proteins (DRP1, MFF and FIS1) was upregulated following treatment with $100 \mu \mathrm{m} \mathrm{FAC} \mathrm{in} \mathrm{a} \mathrm{time-dependent} \mathrm{manner} \mathrm{(Fig.} \mathrm{2B} \mathrm{and} \mathrm{C).}$ In addition, the protein expression of BAX and cytochrome $c$ was upregulated following treatment with $100 \mu \mathrm{m}$ FAC. These results indicated that FAC promoted mitochondrial fission and caused destruction of mitochondrial morphology and leakage 
A
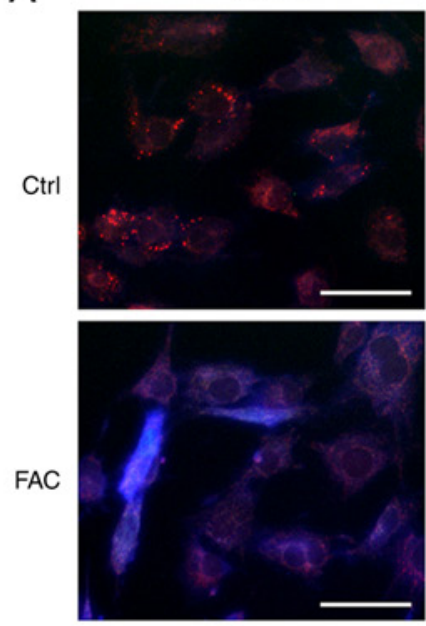

B

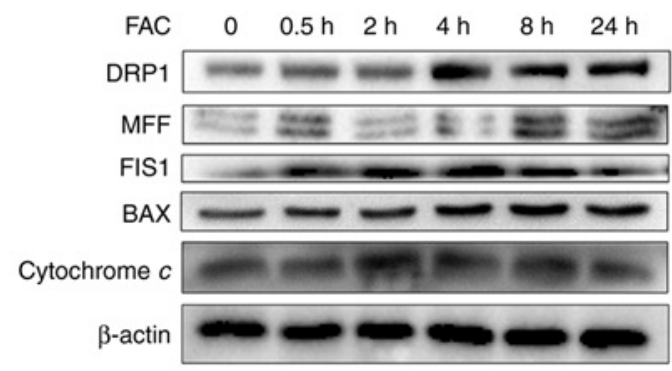

Cytochrome $c$
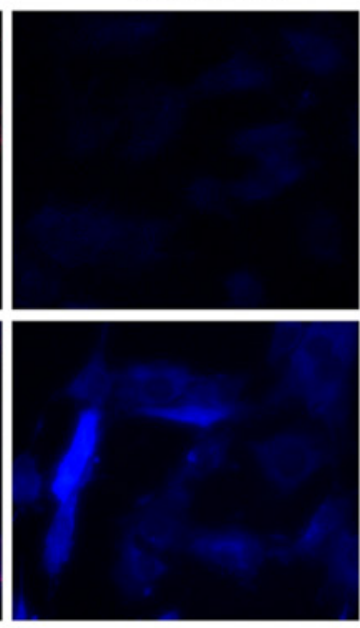

BAX
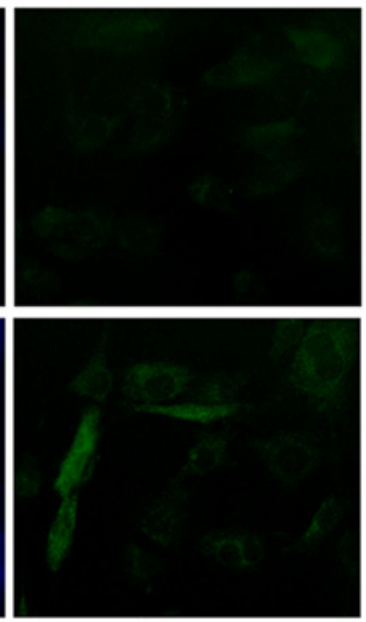

Mito-Tracker
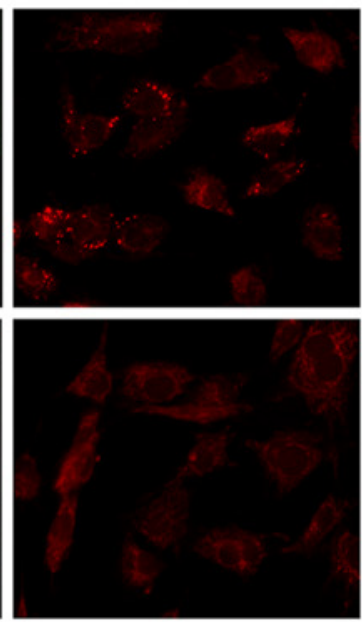
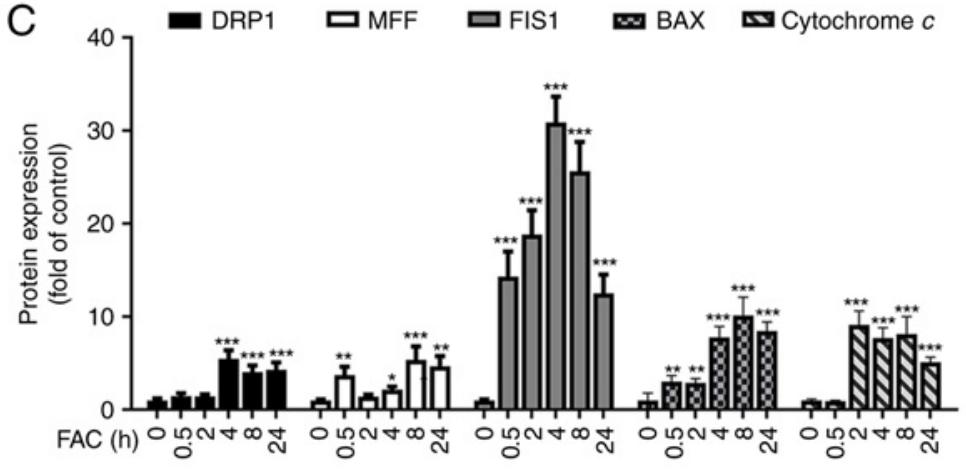

Figure 2. Iron overload impairs mitochondrial dynamics in chondrocytes. (A) Chondrocytes were treated with $100 \mu \mathrm{m}$ FAC for $24 \mathrm{~h}$ and immunofluorescence staining was conducted to examine the expression and localization of BAX (green) and cytochrome $c$ (blue). Mito-Tracker Red was used to stain mitochondria. Scale bar, $50 \mu \mathrm{m}$ (B) Chondrocytes were treated with $100 \mu \mathrm{m}$ FAC for $24 \mathrm{~h}$ and western blotting was conducted to examine the expression of the mitochondrial fission proteins MFF, DRP1, FIS1, BAX and cytochrome $c$. Representative bands of western blots were from the same sample; for FIS1, BAX and cytochrome $c$ proteins, the molecular weights of which were very close, images were derived from three or four gels of the same sample. (C) The band density of DRP1, MFF, FIS1, BAX and cytochrome $c$ was quantified and normalized to the control. Data are presented as mean $\pm \mathrm{SD}$. ${ }^{*} \mathrm{P}<0.05 ;{ }^{* *} \mathrm{P}<0.01 ;{ }^{* * *} \mathrm{P}<0.001 \mathrm{vs} .0 \mathrm{~h}$. MFF, mitochondrial fission factor; DRP1, dynamin-related protein 1; FIS1, mitochondrial fission 1 protein; FAC, ferric ammonium citrate; Ctrl, control group.

of cytochrome $c$, a classic caspase-dependent apoptosis inducer in mitochondria, further promoting mitochondria-dependent apoptosis.

Calcium chelator decreases intracellular iron concentration. Calcium chelator was reported to inhibit iron influx (21). To test this hypothesis, chondrocytes were treated with $100 \mu \mathrm{m} \mathrm{FAC}$, with or without BAPTA-AM. The fluorescence dye calcein-AM was used to assess the ferrous iron uptake and outflow in chondrocytes. As shown in Fig. 3, the fluorescence intensity significantly decreased in the FAC treatment group, indicating elevated iron content in chondrocytes. By contrast, the fluorescence intensity significantly increased in the BAPTA-AM co-treatment group, indicating that the BAPTA-AM co-treatment decreased iron concentration in chondrocytes.

Calcium chelator inhibits ROS production and protects against collapse of mitochondrial membrane potential induced by iron overload. It was reported that excess iron in mitochondria could generate ROS via the Fenton reaction (5). As shown in Fig. 4A, DCFH-DA staining demonstrated that FAC promoted ROS production and this effect was inhibited by the calcium chelator BAPTA-AM. Iron overload impairs normal mitochondrial morphology and function, whereas mitochondrial dysfunction, in turn, affects cellular iron influx and increases ROS production (24). Next, JC-1 staining was utilized to investigate whether calcium chelator could inhibit the collapse of the mitochondrial membrane potential. As shown in Fig. 4B-D, the ratio of green JC-1 monomers to red JC-1 aggregates was increased in the FAC treatment group, while BAPTA-AM treatment suppressed the changes in mitochondrial membrane potential induced by iron. These results indicated that calcium chelators may protect against iron overload-induced ROS production and mitochondrial dysfunction.

Calcium chelator protects chondrocytes against iron overload-induced mitochondrial damage. Mitochondria in healthy chondrocytes display a wire-like shape. As shown in Fig. 5A, FAC treatment $(100 \mu \mathrm{m})$ significantly increased the number of granulated mitochondria in chondrocytes, while BAPTA-AM restored the normal mitochondrial shape in chondrocytes. Mitochondrial fission and fusion are known to regulate the function and morphology of mitochondria. A number of studies have shown that mitochondrial fission is an early event during the process of cell death (25). As shown in Fig. 5A, the expression of the mitochondrial fission proteins, DRP1, MFF and FIS1, was found to be 
A

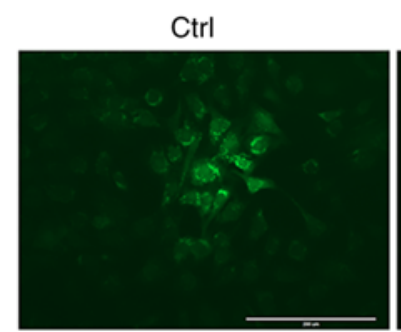

FAC

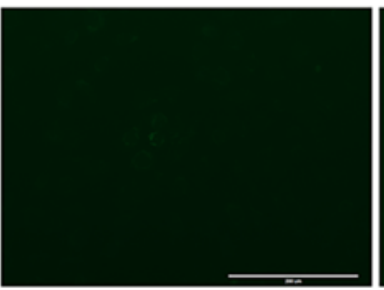

$\mathrm{FAC}+\mathrm{BA}$

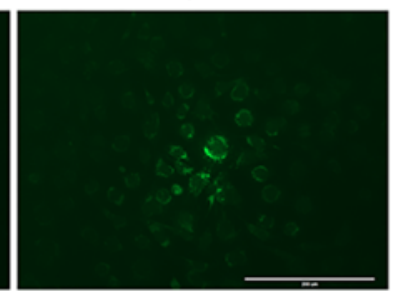

$\mathrm{B}$

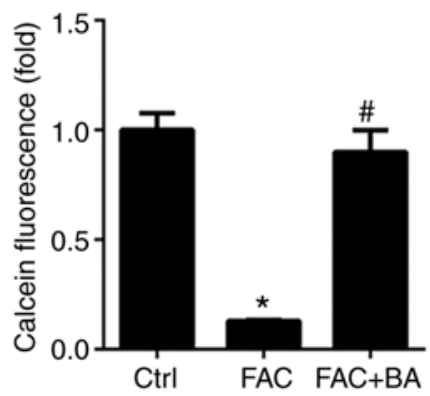

Figure 3. Iron chelator BAPTA-AM inhibits iron influx in chondrocytes. (A) Ferrous iron uptake into chondrocytes was determined by the quenching of calcein fluorescence, which is an indicator of intracellular iron levels. Chondrocytes were treated with $100 \mu \mathrm{m}$ FAC in the presence or absence of $10 \mu \mathrm{m}$ of the calcium chelator BAPTA-AM. Intracellular iron was stained with $0.5 \mathrm{~mm}$ calcein-AM for 15 min and then observed by confocal microscopy. (B) The quenching of green fluorescence represents increased intracellular iron levels. Scale bars, $200 \mu \mathrm{m}$. Data are expressed as the mean $\pm \mathrm{SD}$. ${ }^{*} \mathrm{P}<0.05$ vs. Ctrl; ${ }^{*} \mathrm{P}<0.05 \mathrm{vs}$. FAC treatment group. FAC, ferric ammonium citrate; BAPTA-AM, BAPTA acetoxymethyl ester; Ctrl, control group; FAC + BA, FAC + BAPTA-AM group.

A
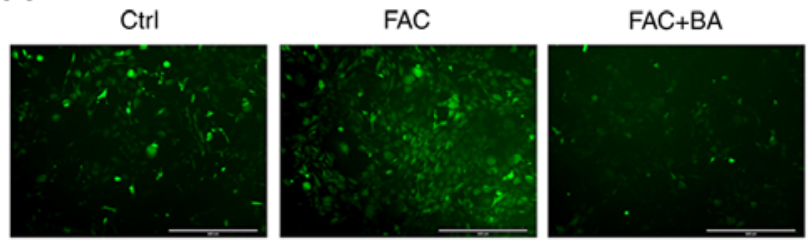

B
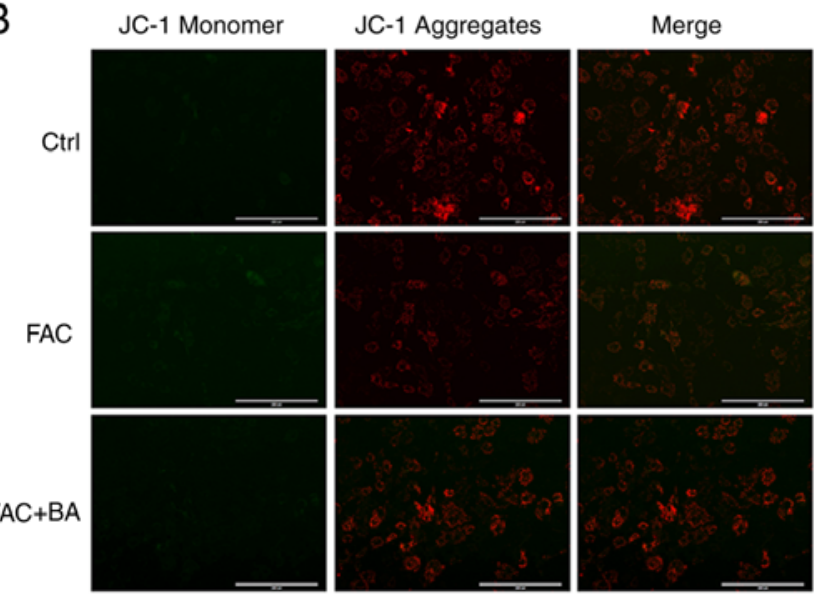

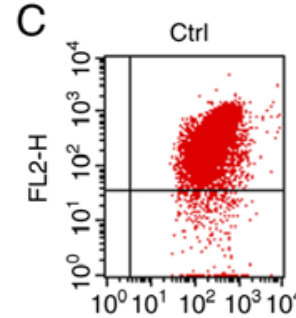

FL1-H

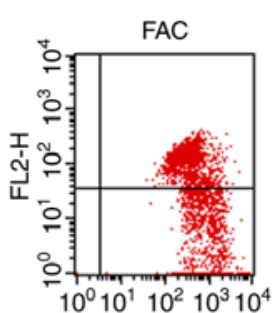

FL1-H

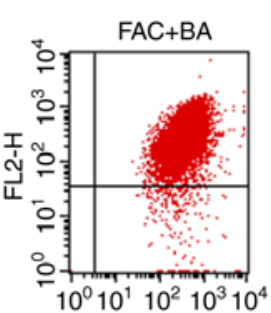

FL1-H

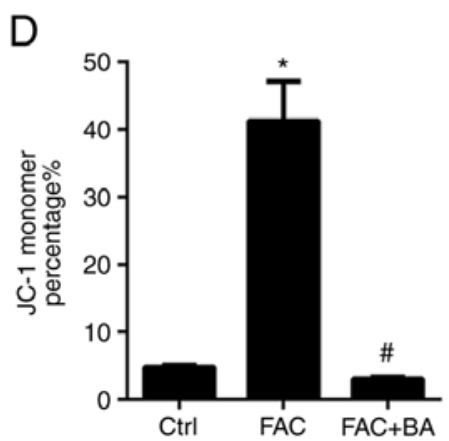

Figure 4. Calcium chelator BAPTA-AM inhibits ROS production and protects against collapse of mitochondrial membrane potential induced by iron overload. (A) Chondrocytes were treated with $100 \mu \mathrm{m}$ FAC in the presence or absence of $10 \mu \mathrm{m}$ of the calcium chelator BAPTA-AM. The quenching of green fluorescence indicates that the intracellular ROS level was reduced in cells treated with BAPTA-AM. Scale bars, $400 \mu \mathrm{m}$. (B) Representative fluorescence microscopy photomicrographs of mitochondrial membrane potential following incubation with JC-1. Red fluorescence indicates healthy mitochondria, whereas green fluorescence indicates mitochondrial dysfunction and mitochondrial membrane potential dissipation. Merged images show colocalization of JC-1 aggregates and monomers. Scale bars, $200 \mu \mathrm{m}$. (C) Representative graphs of flow cytometric analysis of the altered mitochondrial membrane potential following incubation with JC-1 dye. (D) Bar graphs showing the changes in mitochondrial membrane potential detected by flow cytometry. Scale bars, $200 \mu \mathrm{m}$. Data are expressed as the mean \pm SD. ${ }^{*} \mathrm{P}<0.05$ vs. Ctrl; ${ }^{~} \mathrm{P}<0.05$ vs. FAC treatment group. FAC, ferric ammonium citrate; BAPTA-AM, BAPTA acetoxymethyl ester; ROS, reactive oxygen species; Ctrl, control group; FAC + BA, FAC + BAPTA-AM group.

upregulated by treatment with $100 \mu \mathrm{m}$ FAC, and it was decreased by treatment with $10 \mu \mathrm{m}$ BAPTA-AM. Moreover, it was observed that the pro-inflammatory cytokine IL-1 $\beta$ increased FAC-induced mitochondrial fission protein expression, whereas this effect was also reversed by BAPTA-AM. In conclusion, the aforementioned results indicated that iron overload significantly promoted mitochondrial destruction and dysfunction, and calcium chelator treatment could effectively preserve the normal mitochondrial morphology and function.
Calcium chelator protects chondrocytes against iron overload-induced apoptosis and lowers the expression of MMPs. Our previous findings demonstrated the association between iron overload and OA progression (26). In the present study, it was investigated whether calcium chelator treatment could protect chondrocytes against iron overload-induced apoptosis and suppress the expression of MMPs. As shown in Fig. 6A and B, FAC treatment significantly promoted chondrocyte apoptosis, whereas BAPTA-AM decreased the chondrocyte apoptosis rate induced by $100 \mu \mathrm{m}$ FAC. A 
A
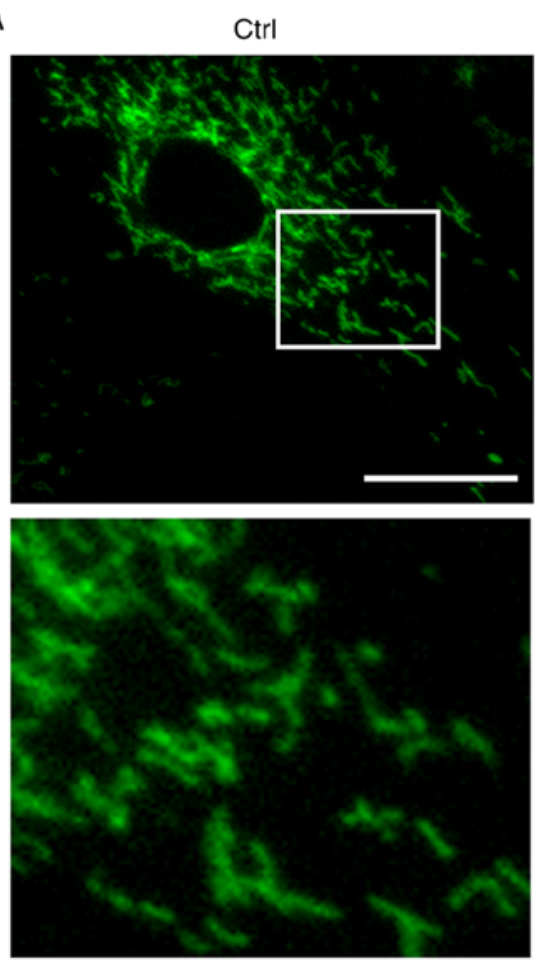

B

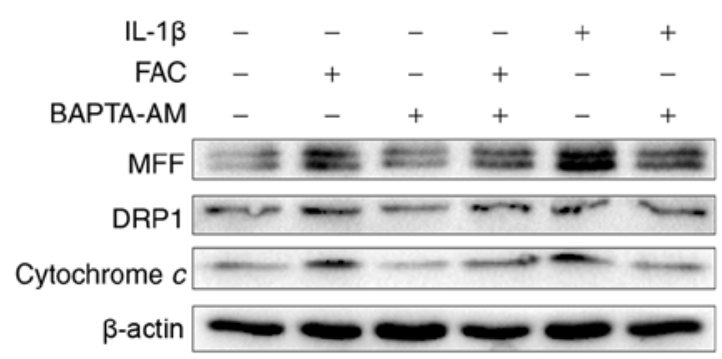

FAC
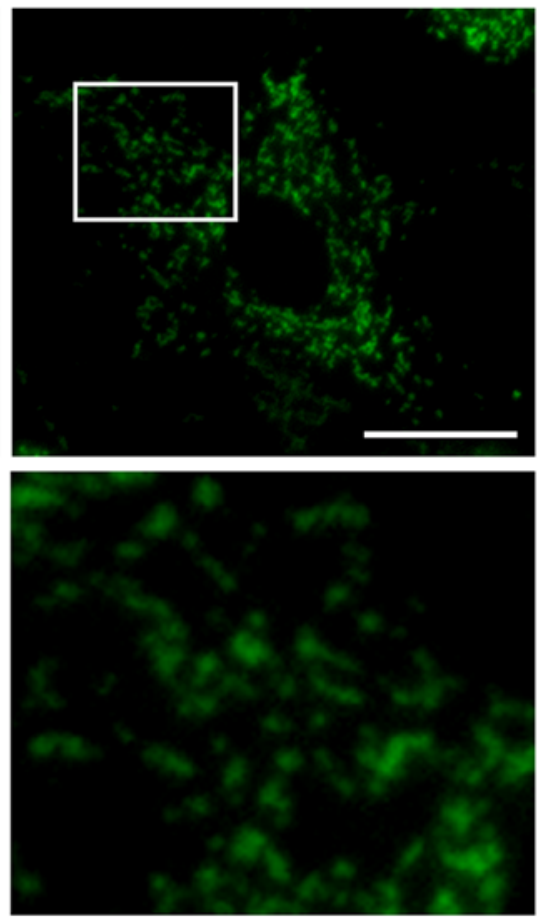

C

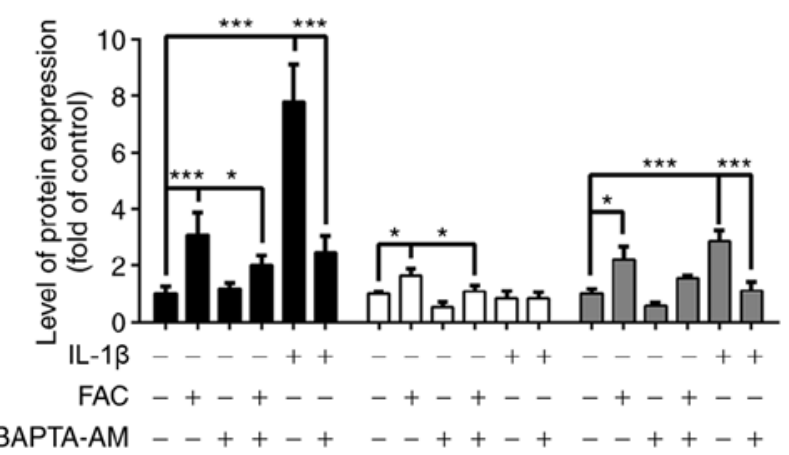

$\mathrm{FAC}+\mathrm{BA}$
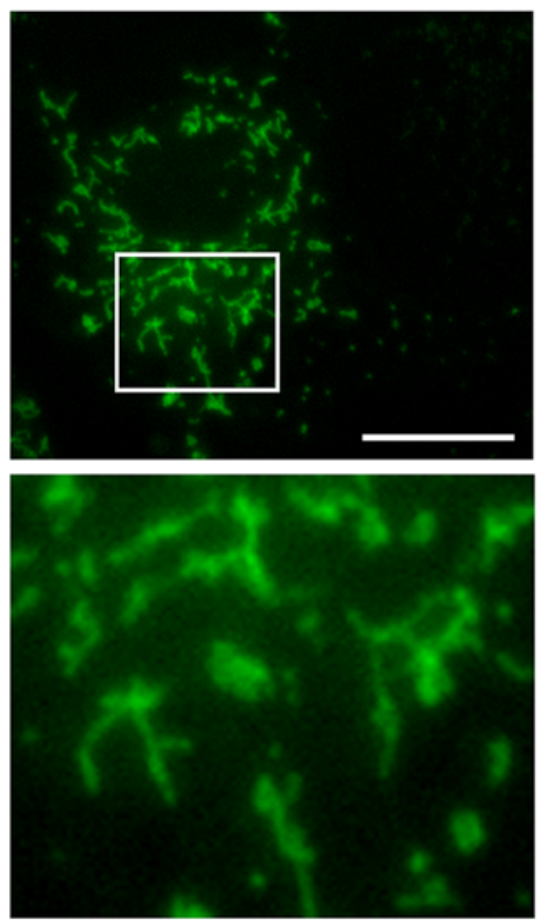

$\square$ FIS1
BAPTA-AM --++-+--++-+--++-+

Figure 5. Calcium chelator BAPTA-AM protects chondrocytes against iron overload-induced mitochondrial damage. (A) Representative fluorescence images of mitochondria and local amplification images of the selected area. Chondrocytes were treated with $100 \mu \mathrm{m}$ FAC in the presence or absence of $10 \mu \mathrm{m}$ of the calcium chelator BAPTA-AM and the morphology of the mitochondria was visualized using Mito-Tracker Green staining. Scale bars, $25 \mu \mathrm{m}$. (B) Chondrocytes were treated with $100 \mu \mathrm{m} \mathrm{FAC}$ or $10 \mathrm{ng} / \mathrm{ml}$ IL-1 $\beta$ in the presence or absence of $10 \mu \mathrm{m}$ of the calcium chelator BAPTA-AM. Western blotting was conducted to examine the protein expression levels of MFF, DRP1 and FIS1. (C) Densitometric analysis of MFF, DRP1 and FIS1 protein expression normalized to $\beta$-actin. Data are presented as mean $\pm \mathrm{SD}$. ${ }^{*} \mathrm{P}<0.05 ;{ }^{* * *} \mathrm{P}<0.001$. MFF, mitochondrial fission factor; DRP1, dynamin-related protein 1; FIS1, mitochondrial fission 1 protein; FAC, ferric ammonium citrate; BAPTA-AM, BAPTA acetoxymethyl ester; Ctrl, control group; FAC + BA, FAC + BAPTA-AM group.

hallmark of OA chondrocytes is the increased production of MMPs. As shown in Fig. 6C and D, BAPTA-AM treatment decreased chondrocyte MMP3 and MMP13 expression, which was induced by $100 \mu \mathrm{m}$ FAC. Moreover, it was observed that FAC and IL-1 $\beta$ co-treatment significantly promoted MMP3 expression compared with the FAC group, and this effect was also inhibited by BAPTA-AM. In summary, these results indicated that calcium chelator treatment may protect chondrocytes against iron overload-induced apoptosis and lower OA-related marker expression.

\section{Discussion}

As the most abundant trace metal in the body, iron participates in a variety of vital functions, including DNA synthesis, oxygen transport, energy metabolism and cellular respiration. However, excess iron accumulation is common in certain tissues or organs, such as the bone marrow, heart and brain, and may lead to the development of pathological conditions or diseases $(12,27)$. By participating in the mitochondrial respiratory chain, iron leads to the generation of ROS through exchanging single electrons with substrates. This triggers oxidative stress, lipid peroxidation and DNA damage, which may result in genomic instability and DNA repair defects, ultimately compromising cell viability and promoting programmed cell death (28). Our previous studies have demonstrated a link between iron overload and OA. Iron overload is common in OA cartilage, as indicated by the presence of hemosiderin deposition. Abnormal cartilage iron accumulation is not only found in hemophilic arthropathy and 
A

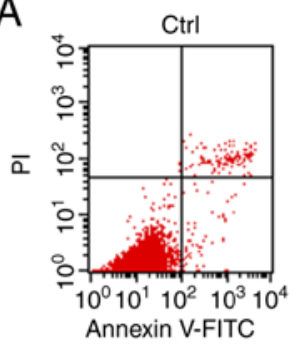

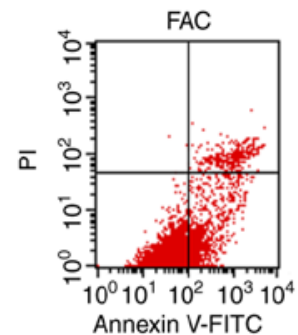

Annexin V-FITC

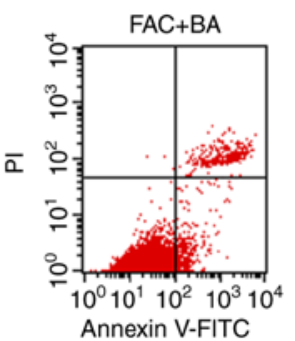

Annexin V-FITC
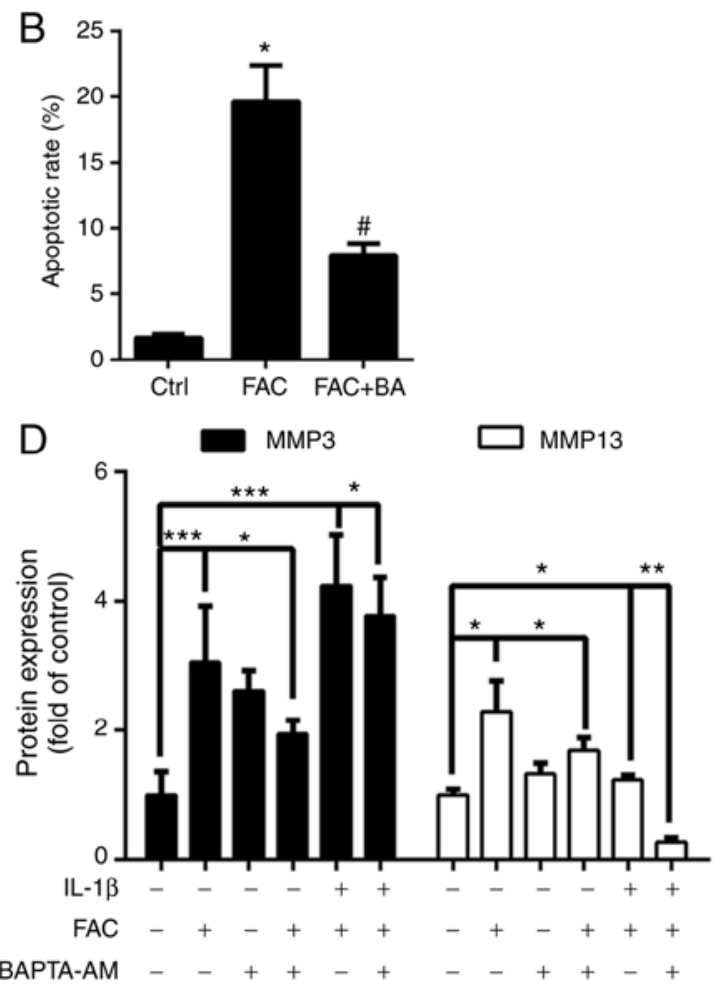

Figure 6. Calcium chelator BAPTA-AM protects chondrocytes against iron overload-induced apoptosis and lowers MMP expression. (A and B) Chondrocytes were treated with $100 \mu \mathrm{m}$ FAC in the presence or absence of $10 \mu \mathrm{m}$ calcium chelator BAPTA-AM for $24 \mathrm{~h}$, and Annexin V-FITC/PI flow cytometric analysis was conducted to detect the apoptotic rate following FAC and BAPTA-AM treatment. Data are presented as mean \pm SD. ${ }^{*} \mathrm{P}<0.05$ vs. Ctrl; ${ }^{*} \mathrm{P}<0.05$ vs. FAC treatment group. (C and D) Chondrocytes were treated with $100 \mu \mathrm{m}$ FAC or $10 \mathrm{ng} / \mathrm{ml} \mathrm{IL-1} \beta$ in the presence or absence of $10 \mu \mathrm{m}$ calcium chelator BAPTA-AM. Western blotting was conducted to examine the protein expression levels of MMP3 and MMP13. (C) Densitometric analysis of MMP3 and MMP13 protein expression normalized to $\beta$-actin. Data are presented as mean $\pm \mathrm{SD} .{ }^{*} \mathrm{P}<0.05 ;{ }^{* *} \mathrm{P}<0.01 ;{ }^{* * *} \mathrm{P}<0.001$. FAC, ferric ammonium citrate; BAPTA-AM, BAPTA acetoxymethyl ester; Ctrl, control group; FAC + BA, FAC + BAPTA-AM group.

hereditary hemochromatosis, but also in traumatic arthritis and rheumatoid arthritis $(29,30)$. A potential cause of increased iron deposition in the afflicted joints of patients with OA may be the microbleeding due to the compromised vasculature, which may result in intra-articular iron loading. Iron chelation is the main treatment of patients with systemic iron overload. Therefore, it is important to investigate new treatment strategies to inhibit iron overload-induced detrimental effects.

Cellular iron homeostasis is delicately regulated by several iron metabolism-related proteins, among which TfR1 plays the most important role in controlling cellular iron levels (31). Chondrocytes take up iron mainly through TfR1 mediated iron-TF-TfR1 complex endocytosis. $\mathrm{Ca}^{2+}$ was reported to regulate iron-TF-tfR1 complex internalization and promote iron influx $(21,32)$. In the present study, the calcium chelator BAPTA-AM was used to test the efficacy of calcium chelator treatment in iron overload-induced chondrocyte damage. BAPTA-AM significantly reduced iron levels in chondrocytes and inhibited iron overload-induced cell apoptosis and the expression of MMPs, thus providing new insights into the treatment of iron overload-induced diseases.

Recently, mitochondria have been shown to play a pivotal role in the progression of OA $(33,34)$. However, the role of mitochondrial dysfunction in iron overload-induced chondrocyte apoptosis and MMP expression remains to be fully elucidated. In the present study, the immunofluorescence results demonstrated that mitochondrial destruction increased along with leakage of cytochrome $c$ from the mitochondria to the cytoplasm and an increase in the expression of BAX. These results indicated that excess iron may lead to disrupted mitochondrial function and morphology. Mitochondrial fission and fusion play important roles in the adaptation of mitochondria to harmful stimuli (35). Mitochondrial fission occurs at the early stages of mitochondrial dysfunction and morphology disruption (36). The results of the present study demonstrated that FAC promoted the expression of mitochondrial fission-related proteins in a time-dependent manner. Mitochondrial morphological assays produced similar results. Following treatment with $100 \mu \mathrm{m}$ FAC for $24 \mathrm{~h}$, the mitochondrial shape changed from a long wire-like to a short, granular shape, while the detrimental effects of iron overload were reversed and the number of wire-like shaped mitochondria was increased following calcium chelator treatment. These results indicated that calcium chelators may protect chondrocytes against iron overload-induced mitochondrial dysfunction.

Iron is required for mitochondria to synthesize heme and iron-sulfur clusters, which are essential for mitochondrial function (37). However, excess iron accumulation in mitochondria may catalyze the generation of highly reactive $\mathrm{OH}$-radicals by reacting with $\mathrm{H}_{2} \mathrm{O}_{2}$ and eventually causing ROS overproduction. Mitochondria are major organelles that generate ROS, but excessive ROS production induces mitochondrial dysfunction. Elevated ROS levels could further destroy mitochondrial structure and function, subsequently activating the mitochondrial apoptotic pathway (38). The results of the present study demonstrated that ROS production was markedly reduced by 
calcium chelator treatment. The results also revealed that FAC treatment decreased mitochondrial membrane potential and lead to mitochondrial membrane depolarization, whereas these effects were notably attenuated by BAPTA-AM treatment.

Oxidative stress has been identified as an important contributor to OA progression. Increased ROS generation does not only activate the mitochondrial apoptotic pathway, but also promotes the expression of MMPs (39). The present study demonstrated that iron reduced cell viability and increased chondrocyte apoptotic rate, which was also reversed by calcium chelator treatment. Our previous study demonstrated that pro-inflammatory cytokines could disrupt iron homeostasis and promote iron influx via promoting the expression of the iron influx regulators TfR1 and DMT1 (26). In the present study, it was observed that the pro-inflammatory cytokine IL-1 $\beta$ promoted mitochondrial fission protein and MMP expression, which was induced by iron overload. This effect was also shown to be inhibited by calcium chelator treatment.

In conclusion, using an in vitro iron overload model, the present study demonstrated that excess iron is toxic to chondrocytes. Iron overload did not only promote chondrocyte apoptosis, but also promoted MMP expression via catalyzing ROS production and mitochondrial dysfunction. Calcium chelator treatment decreased intracellular iron concentration and iron overload-induced ROS overproduction and mitochondrial dysfunction, thus protecting against iron overload-induced chondrocyte apoptosis and MMP expression. These findings indicate that calcium chelators may hold promise in the prevention or treatment of iron overload-related OA progression. This was a preliminary study designed to demonstrate whether a calcium chelator could protect against iron overload-induced chondrocyte degeneration, as compared to iron chelators. There are numerous calcium chelator agents that have been approved by FDA; in addition, the present study is potentially limited by the lack of in vivo experiments, and further studies are required to fully elucidate the exact mechanism underlying the role of the calcium chelator BAPTA-AM in modulating chondrocyte iron influx.

\section{Acknowledgements}

Not applicable.

\section{Funding}

The present study was supported by the National Natural Science Foundation of China (grant no. 82002325) and the Natural Science Foundation of Shandong Province (grant nos. ZR2020QH075 and ZR2020QH264).

\section{Availability of data and materials}

The data that support the findings of this study are available from the corresponding author upon reasonable request.

\section{Authors' contributions}

XJ designed the experiments. XJ and QW wrote the manuscript and analyzed the data. TD, WZ, XL, TL, GW and FC carried out the experiments. QL, TD and QW made substantial contributions to analysis and interpretation of data, supervised the present study, searched the literature and revised the manuscript. XC and TD confirm the authenticity of all the raw data. All the authors have read and approved the final manuscript.

\section{Ethics approval and consent to participate}

All animal protocols were approved by the Institutional Animal Care of the Shandong Provincial Hospital Affiliated to Shandong First Medical University (no. 2020-526).

\section{Patient consent for publication}

Not applicable.

\section{Competing interests}

The authors declare that they have no competing interests.

\section{References}

1. Jing X, Du T, Li T, Yang X, Wang G, Liu X, Jiang Z and Cui X: The detrimental effect of iron on OA chondrocytes: Importance of pro-inflammatory cytokines induced iron influx and oxidative stress. J Cell Mol Med 25: 5671-5680, 2021.

2. Jing X, Lin J, Du T, Jiang Z, Li T, Wang G, Liu X, Cui X and Sun K: Iron overload is associated with accelerated progression of osteoarthritis: The role of DMT1 mediated iron homeostasis. Front Cell Dev Biol 8: 594509, 2020.

3. Jeney V: Clinical impact and cellular mechanisms of iron overload-associated bone loss. Front Pharmacol 8: 77, 2017.

4. Sheth S: Iron chelation: An update. Curr Opin Hematol 21: 179-185, 2014.

5. Gozzelino R and Arosio P: Iron homeostasis in health and disease. Int J Mol Sci 17: 130, 2016.

6. Leipuviene R and Theil E: The family of iron responsive RNA structures regulated by changes in cellular iron and oxygen. Cell Mol Life Sci 64: 2945-2955, 2007.

7. Xie Y, Hou W, Song X, Yu Y, Huang J, Sun X, Kang R and Tang D: Ferroptosis: Process and function. Cell Death Differe 23: 369-379, 2016.

8. Siddique A and Kowdley KV: Review article: The iron overload syndromes. Aliment Pharmacol Ther 35: 876-893, 2012.

9. Ogilvie-Harris DJ and Fornaiser VL: Synovial iron deposition in osteoarthritis and rheumatoid arthritis. J Rheumatol 7: 30-36, 1980.

10. Roosendaal G, Tekoppele JM, Vianen ME, van den Berg HM, Lafeber FP and Bijlsma JW: Articular cartilage is more susceptible to blood induced damage at young than at old age. J Rheumatol 27: 1740-1744, 2000.

11. Naughton DP: Iron(III)-mediated intra-articular crystal deposition in arthritis: A therapeutic role for iron chelators. Med Hypotheses 57: 120-122, 2001.

12. Nakamura T, Naguro I and Ichijo H: Iron homeostasis and iron-regulated ROS in cell death, senescence and human diseases. Biochim Biophys Acta 1863: 1398-1409, 2019.

13. Kennish L, Attur M, Oh C, Krasnokutsky S, Samuels J, Greenberg JD, Huang $X$ and Abramson SB: Age-dependent ferritin elevations and HFE C282Y mutation as risk factors for symptomatic knee osteoarthritis in males: A longitudinal cohort study. BMC Musculoskelet Disord 15: 8, 2014.

14. Suantawee T, Tantavisut S, Adisakwattana S, Tanavalee A, Yuktanandana P, Anomasiri W, Deepaisarnsakul B and Honsawek S: Oxidative stress, vitamin e, and antioxidant capacity in knee osteoarthritis. J Clin Diagn Res 7: 1855-1859, 2013.

15. Blanco FJ, Rego I and Ruiz-Romero C: The role of mitochondria in osteoarthritis. Nat Rev Rheumatol 7: 161-169, 2011.

16. Kühn LC: Iron regulatory proteins and their role in controlling iron metabolism. Metallomics 7: 232-243, 2015.

17. Kumfu S, Chattipakorn SC, Fucharoen S and Chattipakorn N: Dual T-type and L-type calcium channel blocker exerts beneficial effects in attenuating cardiovascular dysfunction in iron-overloaded thalassaemic mice. Exp Physiol 101: 521-539, 2016. 
18. Sripetchwandee J, KenKnight SB, Sanit J, Chattipakorn S and Chattipakorn N: Blockade of mitochondrial calcium uniporter prevents cardiac mitochondrial dysfunction caused by iron overload. Acta Physiol (Oxf) 210: 330-341, 2014.

19. Kumfu S, Khamseekaew J, Palee S, Srichairatanakool S, Fucharoen S, Chattipakorn SC and Chattipakorn N: Combined iron chelator and T-type calcium channel blocker exerts greater efficacy on cardioprotection than monotherapy in iron-overload thalassemic mice. Eur J Pharmacol 822: 43-50, 2018.

20. Zhang Y, Zhao X, Chang Y, Zhang Y, Chu X, Zhang X, Liu Z, Guo H, Wang N, Gao Y, et al: Calcium channel blockers ameliorate iron overload-associated hepatic fibrosis by altering iron transport and stellate cell apoptosis. Toxicol Appl Pharmacol 301: 50-60, 2016

21. Cui R, Choi SE, Kim TH, Lee HJ, Lee SJ, Kang Y, Jeon JY, Kim HJ and Lee KW: Iron overload by transferrin receptor protein 1 regulation plays an important role in palmitate-induced insulin resistance in human skeletal muscle cells. FASEB J 12 : fj201800448R, 2018.

22. Livak KJ and Schmittgen TD: Analysis of relative gene expression data using real-time quantitative PCR and the 2(-Delta Delta C(T)) method. Methods 25: 402-408, 2001.

23. Meyer JN, Leuthner TC and Luz AL: Mitochondrial fusion, fission, and mitochondrial toxicity. Toxicology 391: 42-53, 2017.

24. Wang H, Liu C, Zhao Y and Gao G: Mitochondria regulation in ferroptosis. Eur J Cell Biol 99: 151058, 2019.

25. Tilokani L, Nagashima S, Paupe V and Prudent J: Mitochondrial dynamics: Overview of molecular mechanisms. Essays Biochem 62: 341-360, 2018

26. Jing X, Lin J, Du T, Jiang Z, Li T, Wang G, Liu X, Cui X and Sun K: Iron overload is associated with accelerated progression of osteoarthritis: The role of DMT1 mediated iron homeostasis. Front Cell Dev Biol 8: 594509, 2021.

27. Chung JY, Kim HS and Song J: Iron metabolism in diabetes-induced Alzheimer's disease: A focus on insulin resistance in the brain. Biometals 31: 705-714, 2018.

28. Gao M, Monian P, Pan Q, Zhang W, Xiang J and Jiang X: Ferroptosis is an autophagic cell death process. Cell Res 26: 1021-1032, 2016

29. Nieuwenhuizen L, Schutgens RE, van Asbeck BS, Wenting MJ, van Veghel K, Roosendaal G, Biesma DH and Lafeber FP: Identification and expression of iron regulators in human synovium: Evidence for upregulation in haemophilic arthropathy compared to rheumatoid arthritis, osteoarthritis, and healthy controls. Haemophilia 19: e218-e227, 2013.
30. Camacho A, Simao M, Ea HK, Cohen-Solal M, Richette P Branco J and Cancela ML: Iron overload in a murine model of hereditary hemochromatosis is associated with accelerated progression of osteoarthritis under mechanical stress. Osteoarthritis Cartilage 24: 494-502, 2016.

31. Gammella E, Buratti P, Cairo G and Recalcati S: The transferrin receptor: The cellular iron gate. Metallomics 9: 1367-1375, 2017.

32. Chen MP, Cabantchik ZI, Chan S, Chan GC and Cheung YF: Iron overload and apoptosis of HL-1 cardiomyocytes: Effects of calcium channel blockade. PLoS One 9: e112915, 2014.

33. Lopez de Figueroa P, Lotz MK, Blanco FJ and Carames B: Autophagy activation and protection from mitochondrial dysfunction in human chondrocytes. Arthritis Rheumatol 67: 966-976, 2015.

34. Zhang Z, Xu T, Chen J, Shao Z, Wang K, Yan Y, Wu C, Lin J, Wang $\mathrm{H}$, Gao W, et al: Parkin-mediated mitophagy as a potential therapeutic target for intervertebral disc degeneration. Cell Death Dis 9: 980, 2018.

35. Tsuchiya M, Ichiseki T, Ueda S, Ueda Y, Shimazaki M, Kaneuji A and Kawahara N: Mitochondrial stress and redox failure in steroid-associated osteonecrosis. Int J Med Sci 15: 205-209, 2018

36. Suwanjang W, Wu KL, Prachayasittikul S, Chetsawang B and Charngkaew K: Mitochondrial dynamics impairment in dexamethasone-treated neuronal cells. Neurochem Res 44: 1567-1581, 2019.

37. Anderson CP, Shen M, Eisenstein RS and Leibold EA: Mammalian iron metabolism and its control by iron regulatory proteins. Biochim Biophys Acta 1823: 1468-1483, 2012.

38. Esposito G, Vos M, Vilain S, Swerts J, Valadas JD, Van Meensel S, Schaap O and Verstreken P: Aconitase causes iron toxicity in Drosophila pink1 mutants. PLoS Genet 9: e1003478, 2013.

39. Bresgen N and Eckl PM: Oxidative stress and the homeodynamics of iron metabolism. Biomolecules 5: 808-847, 2015.

This work is licensed under a Creative Commons Attribution-NonCommercial-NoDerivatives 4.0 International (CC BY-NC-ND 4.0) License. 